\title{
Observational constraints on Tsallis modified gravity
}

\author{
Mahnaz Asghari, ${ }^{1 \star}$ Ahmad Sheykhi ${ }^{1} \dagger$ \\ ${ }^{1}$ Physics Department, College of Sciences, Shiraz University, Shiraz 71454, Iran and \\ Biruni Observatory, College of Sciences, Shiraz University, Shiraz 71454, Iran
}

Accepted XXX. Received YYY; in original form ZZZ

\begin{abstract}
The thermodynamics-gravity conjecture reveals that one can derive the gravitational field equations by using the first law of thermodynamics and vice versa. Considering the entropy associated with the horizon in the form of nonextensive Tsallis entropy, $S \sim A^{\beta}$ here we first derive the corresponding gravitational field equations by applying the Clausius relation $\delta Q=T \delta S$ to the horizon. We then construct the Friedmann equations of Friedmann-LemaitreRobertson-Walker (FLRW) universe based on Tsallis modified gravity (TMG). Moreover, in order to constrain the cosmological parameters of TMG model, we use observational data, including Planck cosmic microwave background (CMB), weak lensing, supernovae, baryon acoustic oscillations (BAO), and redshift-space distortions (RSD) data. Numerical results indicate that TMG model with a quintessential dark energy is more compatible with the low redshift measurements of large scale structures by predicting a lower value for the structure growth parameter $\sigma_{8}$ with respect to $\Lambda \mathrm{CDM}$ model. This implies that TMG model would slightly alleviate the $\sigma_{8}$ tension.
\end{abstract}

Key words: cosmology : theory

\section{INTRODUCTION}

Considering the discovery of black hole thermodynamics in 1970 decade (Bardeen et al. 1973; Bekenstein 1973; Hawking 1975), it has been known that there is a remarkable analogy between thermodynamics and gravity. Accordingly, there should be some deep relationship between thermodynamics and gravitational field equations, which first disclosed by Jacobson in 1995 (Jacobson 1995). He derived the covariant form of the Einstein field equations by using the Clausius relation $\delta Q=T \delta S$, together with proportionality of entropy to the horizon area. Jacobson's investigation reveals that Einstein equations of general relativity is nothing but an equation of state for the spacetime. Also there are more studies in order to explore the profound connection between the theory of general relativity and the laws of thermodynamics (Padmanabhan 2002, 2005; Eling et al. 2006; Akbar \& Cai 2006; Paranjape et al. 2006; Padmanabhan \& Paranjape 2007; Akbar \& Cai 2007b; Kothawala et al. 2007; Padmanabhan 2010). Moreover, it is possible to rewrite the Friedmann equations in the form of the first law of thermodynamics at the apparent horizon of FLRW universe and vice versa (Verlinde 2000; Wang et al. 2001; Frolov \& Kofman 2003; Danielsson 2005; Cai \& Kim 2005; Bousso 2005; Akbar \& Cai 2007a; Sheykhi 2010a). The relationship between gravity and thermodynamics is also considered in the context of braneworld cosmology (Calcagni 2005; Sheykhi et al. 2007a,b; Sheykhi \& Wang 2009).

\footnotetext{
* mahnaz.asghari@shirazu.ac.ir
}

$\dagger$ asheykhi@shirazu.ac.ir
Although there are interesting proposals on disclosing the relationship between gravity and thermodynamics, the nature of this deep connection is not clearly explained. To this aim, Verlinde proposed gravity as an entropic force (rather than a fundamental one) caused by a change in the amount of information associated with the positions of material bodies (Verlinde 2011). Following this holographic scenario, he derived the Newton's law of gravity and then extended results to relativistic case which yield the Einstein equations. There are more investigations on Verlinde's entropic force in the literatures (Cai et al. 2010a,b; Myung \& Kim 2010; Sheykhi 2010b; Banerjee \& Majhi 2010; Cai et al. 2010c; Wei 2010; Ho et al. 2010; Konoplya 2010; Kiselev \& Timofeev 2011; Hendi \& Sheykhi 2011b,a; Wei et al. 2011; Sheykhi 2012; Sheykhi \& Teimoori 2012; Sheykhi \& Sarab 2012). On the other hand, Padmanabhan considered spacetime as an emergent structure (rather than a pre-existing background manifold) in addition to treating field equations as the equations of emergent phenomena (Padmanabhan 2012). He discussed the spatial expansion of the universe as emergence of space as cosmic time progresses. One can find related explorations on Padmanabhan's approach in Ref. (Sheykhi et al. 2013b; Tu \& Chen 2013; Ling \& Pan 2013; Eune \& Kim 2013; Sheykhi et al. 2013a; Ai et al. 2013; Tu \& Chen 2015).

In the cosmological setup, it has been shown that one can derive the Friedmann equation of FLRW universe from the first law of thermodynamics $\mathrm{d} E=T \mathrm{~d} S+W \mathrm{~d} V$ at apparent horizon (Akbar \& Cai 2007a). In this relation, $S$ is the entropy associated with the apparent horizon, which has the same expression as the entropy of the black hole in the background gravity theory. The only change needed is replacing 
the black hole horizon radius $r_{+}$with the apparent horizon radius $\tilde{r}_{\mathrm{A}}$ in the entropy associated with the apparent horizon. Accordingly, $T$ is the associated temperature with the apparent horizon, $E$ is the total energy content of the universe and $V$ is the volume inside the apparent horizon, and $W$ is the work density defined as $W=\frac{1}{2}(\rho-p)$, where $\rho$ and $p$ are the energy density and pressure of matter and energy content in the universe, respectively.

The most familiar relation for the black hole entropy is the Bekenstein-Hawking area law entropy (Hawking 1974, 1975)

$S_{B H}=\frac{A}{4 G}$,

where $A=4 \pi r_{+}^{2}$ is the black hole horizon area. It should be noted that area law of black hole entropy does not always hold. For example, two well-known quantum corrections to the area law are logarithmic (arises from the loop quantum gravity) and power-law (appears in dealing with the entanglement of quantum fields inside and outside the horizon) corrections which have been extensively investigated in the literatures (Rovelli 1996; Mann \& Solodukhin 1997; Ashtekar et al. 1998; Kaul \& Majumdar 2000; Das et al. 2002, 2008b; Banerjee \& Majhi 2008a,b; Das et al. 2008a; Zhang 2008; Radicella \& Pavón 2010). On the other hand, it was argued that the Boltzmann-Gibbs (BG) theory is not convincing in divergent partition function systems including large scales gravitational systems (Gibbs 2010). As a result, the BG additive entropy should be generalized to non-additive (non-extensive) entropy for such systems (Tsallis 1988; Lyra \& Tsallis 1998; Tsallis et al. 1998; Tsallis 2011, 2012; da C. Nunes et al. 2014). In this regards, and using the statistical arguments, Tsallis and Cirto argued that the microscopic mathematical expression of the thermodynamical entropy of a black hole does not obey the area law and should be modified as (Tsallis \& Cirto 2013; Sheykhi 2018)

$S=\gamma A^{\beta}$

where $\gamma$ is a constant, $\beta$ is called the non-extensive or Tsallis parameter, and $A$ is the black hole horizon area. It should be noted that in the limit $\beta \rightarrow 1$ the area law entropy expression will be recovered. For more investigations related to Tsallis entropy, refer to e.g. (Sayahian Jahromi et al. 2018; Abdollahi Zadeh et al. 2018; Saridakis et al. 2018; Sadri 2019; Mamon et al. 2020; Ghoshal \& Lambiase 2021; da Silva \& Silva 2021; Nojiri et al. 2021).

In the context of general relativity, one can derive Einstein field equations by applying the area law entropy (1) in Clausius relation (Eling et al. 2006), which describes the concordance $\Lambda$ CDM model. However, in spite of the fact that $\Lambda \mathrm{CDM}$ model is properly confirmed by current observational data (Percival et al. 2010; Hinshaw et al. 2013), there are some discrepancies between the Planck cosmic microwave background (CMB) data (Aghanim et al. 2020), and low redshift observations, chiefly local measurements of the structure growth parameter $\sigma_{8}$ (Allen et al. 2003), and also local determinations of the Hubble constant $H_{0}$ (Riess et al. 2016, 2018, 2019, 2021). These tensions might indicate some new physics beyond the standard cosmological model. In this direction, we investigate the potentiality of Tsallis modified gravity (TMG) in alleviating the mismatch between local observations and high redshift measurements.

In order to derive the modified Friedmann equations from
Tsallis entropy at apparent horizon of FLRW universe, we should consider $A$ in relation (2) as the area of apparent horizon defined as

$A=4 \pi \tilde{r}_{\mathrm{A}}^{2}$,

where $\tilde{r}_{\mathrm{A}}$ is the apparent horizon radius (Bak \& Rey 2000)

$\tilde{r}_{\mathrm{A}}=\left(H^{2}+\frac{K}{a^{2}}\right)^{-1 / 2}$,

in which $H$ is the Hubble parameter and $K=-1,0,1$ is the curvature constant corresponding to open, flat and closed universe, respectively. Throughout this paper we set $k_{\mathrm{B}}=c=$ $\hbar=1$ for simplicity.

The paper is organized as follows. In section 2, we derive the corresponding field equations as well as the modified Friedmann equations in the context of TMG model. Section 3 is dedicated to numerical solutions of TMG model. In section 4 we constrain our model with observational data. We summarize our results in section 5 .

\section{MODIFIED GRAVITATIONAL FIELD EQUATIONS FROM TSALLIS ENTROPY}

In this approach, we consider a spatially flat homogeneous and isotropic FLRW universe, described by the following line element in the background level

$\mathrm{d} s^{2}=a^{2}(\tau)\left(-\mathrm{d} \tau^{2}+\mathrm{d} \boldsymbol{x}^{2}\right)$,

where $\tau$ is the conformal time. Moreover, we are interested in scalar perturbations to linear order, so following Ref. (Ma \& Bertschinger 1995), the perturbed metric in conformal Newtonian gauge is

$\mathrm{d} s^{2}=a^{2}(\tau)\left(-(1+2 \Psi) \mathrm{d} \tau^{2}+(1-2 \Phi) \mathrm{d} \boldsymbol{x}^{2}\right)$,

where $\Psi$ and $\Phi$ are gravitational potentials. Also in synchronous gauge we have

$\mathrm{d} s^{2}=a^{2}(\tau)\left(-\mathrm{d} \tau^{2}+\left(\delta_{i j}+h_{i j}\right) \mathrm{d} x^{i} \mathrm{~d} x^{j}\right)$,

in which $h_{i j}=\operatorname{diag}(-2 \eta,-2 \eta, h+4 \eta)$, with scalar perturbations $h$ and $\eta$. We assume the matter and energy content of the universe as a perfect fluid with the following energymomentum tensor

$T_{\mu \nu}=(\rho+p) u_{\mu} u_{\nu}+g_{\mu \nu} p$,

where $\rho=\bar{\rho}+\delta \rho$ is the energy density, $p=\bar{p}+\delta p$ is the pressure, and $u_{\mu}$ is the four-velocity (and a bar indicates the background level).

In order to derive Einstein field equations from Tsallis entropy, we apply the Clausius relation

$\delta Q=T \delta S$.

It is assumed that the Clausius relation is satisfied on a local causal horizon $\mathcal{H}$ (which here it is the apparent horizon).

Let us now consider equation (9) more specifically. The associated temperature with the apparent horizon is given by

$T=\frac{\kappa}{2 \pi}$,

where $\kappa$ is the surface gravity at apparent horizon. Also, ac- 
cording to equation (2), $\delta S$ can be written as

$\delta S=\gamma \beta A^{\beta-1} \delta A$.

In order to define $\delta Q$, we follow the approach of Ref. (Jacobson 1995; Eling et al. 2006) which reads

$\delta Q=-\kappa \int_{\mathcal{H}} \lambda T_{\mu \nu} k^{\mu} k^{\nu} \mathrm{d} \lambda \mathrm{d} A$,

and also we have

$\delta A=\int_{\mathcal{H}} \theta \mathrm{d} \lambda \mathrm{d} A$

$\theta=-\lambda R_{\mu \nu} k^{\mu} k^{\nu}$.

Substituting relations (10),(11) and (12) in equation (9) would result in

$\kappa \int_{\mathcal{H}}(-\lambda) T_{\mu \nu} k^{\mu} k^{\nu} \mathrm{d} \lambda \mathrm{d} A$

$=\frac{\kappa}{2 \pi} \gamma \beta \int_{\mathcal{H}}(-\lambda) R_{\mu \nu} k^{\mu} k^{\nu} A^{\beta-1} \mathrm{~d} \lambda \mathrm{d} A$,

$\rightarrow \int_{\mathcal{H}}(-\lambda)\left(-2 \pi T_{\mu \nu}+\gamma \beta R_{\mu \nu} A^{\beta-1}\right) k^{\mu} k^{\nu} \mathrm{d} \lambda \mathrm{d} A=0$.

So for all null $k^{\mu}$ we should have

$-2 \pi T_{\mu \nu}+\gamma \beta R_{\mu \nu} A^{\beta-1}=f g_{\mu \nu}$,

in which $f$ is a scalar. Then, the energy-momentum conservation $\left(\nabla^{\mu} T_{\mu \nu}=0\right)$ would impose

$\nabla^{\mu}\left(\gamma \beta R_{\mu \nu} A^{\beta-1}-f g_{\mu \nu}\right)=0$,

which after doing some calculations results in

$\frac{1}{2} \gamma \beta\left(\partial_{\nu} R\right) A^{\beta-1}+\gamma \beta R_{\mu \nu} \partial^{\mu} A^{\beta-1}=\partial_{\nu} f$.

However, the LHS of equation (19) is not the gradient of a scalar. It reveals that the Clausius relation does not hold due to non-equilibrium thermodynamics. Hence, in order to resolve this contradiction, we use the entropy balance relation (Eling et al. 2006)

$\delta S=\frac{\delta Q}{T}+\mathrm{d}_{i} S$,

where $\mathrm{d}_{i} S$ is the entropy produced inside the system due to irreversible processes (De Groot \& Mazur 1962). In order to restore the energy-momentum conservation, we choose $\mathrm{d}_{i} S$ as

$\mathrm{d}_{i} S=\gamma \beta \int_{\mathcal{H}}(-\lambda) \nabla_{\mu} \nabla_{\nu} A^{\beta-1} k^{\mu} k^{\nu} \mathrm{d} \lambda \mathrm{d} A$.

Thus, equation (20) reads

$$
\begin{aligned}
& \gamma \beta \int_{\mathcal{H}}(-\lambda) R_{\mu \nu} k^{\mu} k^{\nu} A^{\beta-1} \mathrm{~d} \lambda \mathrm{d} A \\
& =2 \pi \int_{\mathcal{H}}(-\lambda) T_{\mu \nu} k^{\mu} k^{\nu} \mathrm{d} \lambda \mathrm{d} A \\
& +\gamma \beta \int_{\mathcal{H}}(-\lambda) \nabla_{\mu} \nabla_{\nu} A^{\beta-1} k^{\mu} k^{\nu} \mathrm{d} \lambda \mathrm{d} A, \\
& \rightarrow \int_{\mathcal{H}}(-\lambda)\left(\gamma \beta R_{\mu \nu} A^{\beta-1}-2 \pi T_{\mu \nu}-\gamma \beta \nabla_{\mu} \nabla_{\nu} A^{\beta-1}\right) \\
& \quad \times k^{\mu} k^{\nu} \mathrm{d} \lambda \mathrm{d} A=0 .
\end{aligned}
$$

Likewise, for all null $k^{\mu}$ we obtain

$\gamma \beta R_{\mu \nu} A^{\beta-1}-2 \pi T_{\mu \nu}-\gamma \beta \nabla_{\mu} \nabla_{\nu} A^{\beta-1}=f g_{\mu \nu}$.

According to the energy-momentum conservation, we should have

$\nabla^{\mu}\left(\gamma \beta R_{\mu \nu} A^{\beta-1}-\gamma \beta \nabla_{\mu} \nabla_{\nu} A^{\beta-1}-f g_{\mu \nu}\right)=0$.

Then, considering $\nabla^{\mu} \nabla_{\mu} \nabla_{\nu} A^{\beta-1}=\nabla_{\nu} \square A^{\beta-1}+R_{\zeta \nu} \partial^{\zeta} A^{\beta-1}$, we get

$\gamma \beta\left(\frac{1}{2}\left(\partial_{\nu} R\right) A^{\beta-1}-\partial_{\nu} \square A^{\beta-1}\right)=\partial_{\nu} f$.

It is possible to define the scalar $\mathcal{L}$ as $\mathcal{L}=R A^{\beta-1}$, which results in

$\frac{\partial \mathcal{L}}{\partial R}=A^{\beta-1}$,
$\partial_{\nu} R=\frac{\partial R}{\partial x^{\nu}}=\frac{\partial R}{\partial \mathcal{L}} \frac{\partial \mathcal{L}}{\partial x^{\nu}}=A^{1-\beta} \partial_{\nu} \mathcal{L}$,
$\rightarrow\left(\partial_{\nu} R\right) A^{\beta-1}=\partial_{\nu} \mathcal{L}$.

Thus equation (26) takes the form

$\gamma \beta\left(\frac{1}{2} \partial_{\nu} \mathcal{L}-\partial_{\nu} \square A^{\beta-1}\right)=\partial_{\nu} f$,

which results in the following equation for the scalar $f$

$$
\begin{aligned}
f & =\gamma \beta\left(\frac{1}{2} \mathcal{L}-\square A^{\beta-1}\right) \\
& =\gamma \beta\left(\frac{1}{2} R A^{\beta-1}-\square A^{\beta-1}\right) .
\end{aligned}
$$

Therefore, the gravitational field equations based on nonextensive Tsallis entropy (2) take the following form

$$
\begin{aligned}
& R_{\mu \nu} A^{\beta-1}-\nabla_{\mu} \nabla_{\nu} A^{\beta-1}-\frac{1}{2} R A^{\beta-1} g_{\mu \nu}+\square A^{\beta-1} g_{\mu \nu} \\
& =\frac{2 \pi}{\gamma \beta} T_{\mu \nu} .
\end{aligned}
$$

Equation (30) is indeed the modified Einstein field equations, when the entropy associated with the horizon does not obey the area law and instead is given by equation (2). When $\beta=1$, equation (30) restores the standard Einstein equations. Given the modified Einstein equations at hand, we are in the position to derive the corresponding modified Friedmann equations in the background of a flat FLRW universe. According to relations (3) and (4), for a spatially flat universe we have

$A^{\beta-1}=(4 \pi)^{\beta-1}\left(\frac{a^{\prime}}{a^{2}}\right)^{2-2 \beta}=(4 \pi)^{\beta-1} H^{2-2 \beta}$,

where a prime indicates a deviation with respect to the conformal time, and $H=a^{\prime} / a^{2}$ is the Hubble parameter. The (00) and ( $i$ i) components of the modified field equations (30) in a background level are given by

$$
\begin{aligned}
& \left(\frac{a^{\prime}}{a^{2}}\right)^{2-2 \beta}\left\{\left(\frac{a^{\prime}}{a}\right)^{2}+2(1-\beta)\left(\frac{a^{\prime \prime}}{a}-2\left(\frac{a^{\prime}}{a}\right)^{2}\right)\right\} \\
& =\frac{8 \pi G}{3} a^{2} \sum_{i} \bar{\rho}_{i}
\end{aligned}
$$




$$
\begin{aligned}
& \left(\frac{a^{\prime}}{a^{2}}\right)^{2-2 \beta}\left\{-2 \frac{a^{\prime \prime}}{a}+\left(\frac{a^{\prime}}{a}\right)^{2}+2(1-\beta)\left[5 \frac{a^{\prime \prime}}{a}-4\left(\frac{a^{\prime}}{a}\right)^{2}\right.\right. \\
& \left.\left.-\frac{a^{\prime \prime \prime}}{a^{\prime}}-(1-2 \beta)\left(\frac{a^{\prime \prime}}{a^{\prime}}-2 \frac{a^{\prime}}{a}\right)^{2}\right]\right\}=8 \pi G a^{2} \sum_{i} \bar{p}_{i},
\end{aligned}
$$

where we have taken the energy momentum tensor $T_{\mu \nu}$ as defined in equation (8), and also we have defined $\gamma=$ $(4 \pi)^{1-\beta} /(4 G \beta)$. Here $i$ indicates the component $i^{t h}$ of the energy in the universe. In terms of the Hubble parameter $H$, the above equations can be written as

$$
\begin{aligned}
& H^{4-2 \beta}+2(1-\beta) H^{2-2 \beta} H^{\prime} \frac{1}{a}=\frac{8 \pi G}{3} \sum_{i} \bar{\rho}_{i}, \\
& H^{2-2 \beta}\left\{2(\beta-2) H^{\prime} \frac{1}{a}-3 H^{2}\right. \\
& \left.-2(1-\beta)\left((1-2 \beta) \frac{H^{\prime 2}}{H^{2}}+\frac{H^{\prime \prime}}{H}\right) \frac{1}{a^{2}}\right\}=8 \pi G \sum_{i} \bar{p}_{i} .
\end{aligned}
$$

Combining equations (34) and (35), one can rewrite the first modified Friedmann equation as

$H^{4-2 \beta}=\frac{1}{4 \beta-3} \frac{8 \pi G}{3} \sum_{i} \bar{\rho}_{i}$.

Taking into account the definition of the total density parameter $\Omega_{\text {tot }}=\bar{\rho} / \rho_{\text {cr }}$, where $\rho_{\text {cr }}=3 H^{2} /(8 \pi G)$ and $\bar{\rho}=\sum_{i} \bar{\rho}_{i}$, we can further rewrite equation (36) in the form

$\Omega_{\mathrm{tot}}=(4 \beta-3) H^{2-2 \beta}$,

which differs from the standard cosmology, unless for the case $\beta=1$ where we have $\Omega_{\text {tot }}=1$.

It should be noted that in this model $\rho_{\text {tot }}$ includes radiation $(\mathrm{R})$, matter $(\mathrm{M})$ (dark matter (DM) and baryons $(\mathrm{B})$ ) and the fluid "f" with a constant equation of state $w_{\mathrm{f}}=\bar{p}_{\mathrm{f}} / \bar{\rho}_{\mathrm{f}}$. Considering the total equation of state $w_{\text {tot }}=\bar{p}_{\text {tot }} / \bar{\rho}_{\text {tot }}$, under the condition $w_{\text {tot }}<(1-2 \beta) / 3$, the universe would experience an accelerated expansion. Choosing $\beta=1$, results in $w_{\text {tot }}<-1 / 3$ which is the condition for an accelerated universe in standard cosmology. However, different values of Tsallis parameter $\beta$, can result in different conditions from general relativity. In particular, considering $\beta \geq 1 / 2$ would result in $w_{\text {tot }}<0$, while for $\beta<1 / 2$, $w_{\text {tot }}$ can take positive value and we still have the late-time acceleration of the cosmic expansion. For example, by choosing $\beta=1 / 3$ we obtain $w_{\text {tot }}<1 / 9$. This implies that one may consider a universe filled with baryonic matter, and still enjoys an accelerated expansion without invoking any dark companion for its matter/energy content. This is consistent with the argument given in (Sheykhi 2020).

Moreover, modified field equations to linear order of perturbations in conformal Newtonian gauge (con) take the form

$$
\begin{aligned}
& \left(\frac{a^{\prime}}{a^{2}}\right)^{2-2 \beta}\left\{3 \frac{a^{\prime}}{a} \Phi^{\prime}+k^{2} \Phi+3\left(\frac{a^{\prime}}{a}\right)^{2} \Psi\right. \\
& \left.+3(1-\beta)\left(\frac{a^{\prime \prime}}{a^{\prime}}-2 \frac{a^{\prime}}{a}\right)\left(\Phi^{\prime}+2 \Psi \frac{a^{\prime}}{a}\right)\right\} \\
& =-4 \pi G a^{2} \sum_{i} \delta \rho_{i \text { (con })},
\end{aligned}
$$

$$
\begin{aligned}
& \left(\frac{a^{\prime}}{a^{2}}\right)^{2-2 \beta}\left\{k^{2} \Phi^{\prime}+\frac{a^{\prime}}{a} k^{2} \Psi+(1-\beta)\left(\frac{a^{\prime \prime}}{a^{\prime}}-2 \frac{a^{\prime}}{a}\right) k^{2} \Psi\right\} \\
& =4 \pi G a^{2} \sum_{i}\left(\bar{\rho}_{i}+\bar{p}_{i}\right) \theta_{i(\mathrm{con})}, \\
& \Phi=\Psi, \\
& \left(\frac{a^{\prime}}{a^{2}}\right)^{2-2 \beta}\left\{2 \frac{a^{\prime \prime}}{a} \Psi-\left(\frac{a^{\prime}}{a}\right)^{2} \Psi+\frac{a^{\prime}}{a} \Psi^{\prime}+2 \frac{a^{\prime}}{a} \Phi^{\prime}+\Phi^{\prime \prime}\right. \\
& +\frac{k^{2}}{3}(\Phi-\Psi)+(1-\beta)\left\{2\left(\frac{a^{\prime \prime}}{a^{\prime}}-2 \frac{a^{\prime}}{a}\right)\left(2 \frac{a^{\prime}}{a} \Psi+\Phi^{\prime}\right)\right. \\
& +2 \Psi\left[(1-2 \beta)\left(\frac{a^{\prime \prime}}{a^{\prime}}-2 \frac{a^{\prime}}{a}\right)^{2}+\frac{a^{\prime \prime \prime}}{a^{\prime}}-7 \frac{a^{\prime \prime}}{a}+8\left(\frac{a^{\prime}}{a}\right)^{2}\right] \\
& \left.\left.+\Psi^{\prime}\left(\frac{a^{\prime \prime}}{a^{\prime}}-2 \frac{a^{\prime}}{a}\right)\right\}\right\}=4 \pi G a^{2} \sum_{i} \delta p_{i(\mathrm{con})} \cdot
\end{aligned}
$$

Also in synchronous gauge (syn) we have

$$
\begin{aligned}
& \left(\frac{a^{\prime}}{a^{2}}\right)^{2-2 \beta}\left\{\frac{a^{\prime}}{a} h^{\prime}-2 k^{2} \eta+(1-\beta) h^{\prime}\left(\frac{a^{\prime \prime}}{a^{\prime}}-2 \frac{a^{\prime}}{a}\right)\right\} \\
& =8 \pi G a^{2} \sum_{i} \delta \rho_{i(\mathrm{syn})} \\
& \left(\frac{a^{\prime}}{a^{2}}\right)^{2-2 \beta} k^{2} \eta^{\prime}=4 \pi G a^{2} \sum_{i}\left(\bar{\rho}_{i}+\bar{p}_{i}\right) \theta_{i(\mathrm{syn})}, \\
& \left(\frac{a^{\prime}}{a^{2}}\right)^{2-2 \beta}\left\{\frac{1}{2} h^{\prime \prime}+3 \eta^{\prime \prime}+\frac{a^{\prime}}{a} h^{\prime}+6 \frac{a^{\prime}}{a} \eta^{\prime}-k^{2} \eta\right. \\
& \left.+2(1-\beta)\left(\frac{a^{\prime \prime}}{a^{\prime}}-2 \frac{a^{\prime}}{a}\right)\left(\frac{1}{2} h^{\prime}+3 \eta^{\prime}\right)\right\}=0, \\
& \left(\frac{a^{\prime}}{a^{2}}\right)^{2-2 \beta}\left\{-2 \frac{a^{\prime}}{a} h^{\prime}-h^{\prime \prime}+2 k^{2} \eta\right. \\
& \left.-2(1-\beta)\left(\frac{a^{\prime \prime}}{a^{\prime}}-2 \frac{a^{\prime}}{a}\right) h^{\prime}\right\}=24 \pi G a^{2} \sum_{i} \delta p_{i(\mathrm{syn})} .
\end{aligned}
$$

It can be easily seen that for $\beta=1$, the standard field equations in Einstein gravity will be restored. In the following we study TMG model in synchronous gauge.

Furthermore, the conservation equations in TMG model are the same as those in general relativity, so for the matter and the fluid components we find the following conservation equations in the synchronous gauge

$$
\begin{aligned}
& \delta_{\mathrm{M}(\mathrm{syn})}^{\prime}=-\theta_{\mathrm{M}(\mathrm{syn})}-\frac{1}{2} h^{\prime}, \\
& \theta_{\mathrm{M}(\mathrm{syn})}^{\prime}=-\frac{a^{\prime}}{a} \theta_{\mathrm{M}(\mathrm{syn})}, \\
& \delta_{\mathrm{f}(\mathrm{syn})}^{\prime}=-3 \frac{a^{\prime}}{a}\left(c_{s, \mathrm{f}}^{2}-w_{\mathrm{f}}\right) \delta_{\mathrm{f}(\mathrm{syn})}-\frac{1}{2} h^{\prime}\left(1+w_{\mathrm{f}}\right) \\
& -\left(1+w_{\mathrm{f}}\right)\left(1+9\left(\frac{a^{\prime}}{a}\right)^{2}\left(c_{s, \mathrm{f}}^{2}-c_{a, \mathrm{f}}^{2}\right) \frac{1}{k^{2}}\right) \theta_{\mathrm{f}(\mathrm{syn})},
\end{aligned}
$$


$\theta_{\mathrm{f}(\mathrm{syn})}^{\prime}=\frac{a^{\prime}}{a}\left(3 c_{s, \mathrm{f}}^{2}-1\right) \theta_{\mathrm{f}(\mathrm{syn})}+\frac{k^{2} c_{s, \mathrm{f}}^{2}}{1+w_{\mathrm{f}}} \delta_{\mathrm{f}(\mathrm{syn})}$.

In order to understand the properties of TMG model more precisely and also constrain the Tsallis parameter $\beta$ with observational data, we modify the Boltzmann code Cosmic Linear Anisotropy Solving System (CLASS) (Blas et al. 2011) according to this model. Furthermore, running a Markov Chain Monte Carlo (MCMC) analysis through the Monte Python code (Audren et al. 2013; Brinckmann \& Lesgourgues 2018), provides us with constraints on the cosmological parameters.

\section{NUMERICAL RESULTS}

In this part, we study TMG model by implementing modified field equations in the CLASS code. Accordingly, we use Planck 2018 data (Aghanim et al. 2020) for the cosmological parameters given by $\Omega_{\mathrm{B}, 0} h^{2}=0.02242, \Omega_{\mathrm{DM}, 0} h^{2}=0.11933$, $H_{0}=67.66 \mathrm{~km} \mathrm{~s}^{-1} \mathrm{Mpc}^{-1}, A_{s}=2.105 \times 10^{-9}$, and $\tau_{\text {reio }}=$ 0.0561 . Since the nature of fluid is unknown to us, without loss of generality we can consider $w_{\mathrm{f}}=-0.98$ and $c_{s, \mathrm{f}}^{2}=1$.

The CMB temperature anisotropy and matter power spectra diagrams are shown in Fig. 1. According to matter power spectra diagrams, there is a decrease in structure growth for TMG model with $\beta<1$. So regarding discrepancies between low redshift observational data and $\mathrm{CMB}$ results, it can be concluded that TMG model with $\beta<1$ is more consistent with local measurements of the structure growth parameter $\sigma_{8}$. This feature of TMG model can be also understood from matter density contrast diagrams in Fig. 2.

It is also interesting to investigate the evolution of the Hubble parameter in TMG model which is described in equation (36). Considering the evolution of Hubble parameter illustrated in Fig. 3, it can be understood that choosing $\beta>1$ would increase the current value of Hubble parameter, which is consequently more compatible with local determinations of this parameter. However, the $H_{0}$ tension problem becomes more severe for $\beta<1$.

\section{OBSERVATIONAL CONSTRAINTS}

In order to explore constraints on cosmological parameters of TMG model, we run an MCMC analysis using the MonTE Python code (Audren et al. 2013; Brinckmann \& Lesgourgues 2018). The following set of parameters is considered in the MCMC analysis:

$\left\{100 \Omega_{\mathrm{B}, 0} h^{2}, \Omega_{\mathrm{DM}, 0} h^{2}, 100 \theta_{s}, \ln \left(10^{10} A_{s}\right), n_{s}, \tau_{\text {reio }}, w_{\mathrm{f}}, \beta\right\}$, where $\Omega_{\mathrm{B}, 0} h^{2}$ and $\Omega_{\mathrm{DM}, 0} h^{2}$ are the baryon and cold dark matter densities relative to the critical density respectively, $\theta_{s}$ is the ratio of the sound horizon to the angular diameter distance at decoupling, $A_{s}$ is the amplitude of the primordial scalar perturbation spectrum, $n_{s}$ is the scalar spectral index, $\tau_{\text {reio }}$ is the optical depth to reionization, $w_{\mathrm{f}}$ is the fluid equation of state parameter, and $\beta$ is the Tsallis parameter. Furthermore, we have four derived parameters which are the reionization redshift $\left(z_{\text {reio }}\right)$, the matter density parameter $\left(\Omega_{\mathrm{M}, 0}\right)$, the Hubble constant $\left(H_{0}\right)$, and the root-mean-square mass fluctuations on scales of $8 h^{-1} \mathrm{Mpc}\left(\sigma_{8}\right)$. According to preliminary numerical works, we consider the prior range $[0.98,1.008]$ for the Tsallis parameter, and also we set no prior range on the fluid equation of state parameter $w_{\mathrm{f}}$.
Table 1. Best fit values and $68 \%$ and $95 \%$ confidence limits for cosmological parameters from "Planck + Planck-SZ + CFHTLenS + Pantheon + BAO + BAORSD" data set for $\Lambda$ CDM and TMG model.

\begin{tabular}{|c|c|c|c|c|}
\hline \multirow[b]{2}{*}{ parameter } & \multicolumn{2}{|r|}{$\Lambda \mathrm{CDM}$} & \multicolumn{2}{|r|}{ TMG model } \\
\hline & best fit & $68 \%$ \& $95 \%$ limits & best fit & $68 \%$ \& $95 \%$ limits \\
\hline $100 \Omega_{\mathrm{B}, 0} h^{2}$ & 2.261 & $2.263_{-0.013-0.025}^{+0.012+0.026}$ & 2.272 & $2.268_{-0.015-0.028}^{+0.014+0.027}$ \\
\hline$\Omega_{\mathrm{DM}, 0} h^{2}$ & 0.1163 & $0.1164_{-0.00079-0.0015}^{+0.00078+0.0015}$ & 0.1166 & $0.1160_{-0.00077-0.0017}^{+0.00097+0.0016}$ \\
\hline $100 \theta_{s}$ & 1.042 & $1.042_{-0.00026-0.00053}^{+0.00029+0.00055}$ & 1.042 & $1.042_{-0.00028-0.00052}^{+0.00026+0.00054}$ \\
\hline $\ln \left(10^{10} A_{s}\right)$ & 3.034 & $3.024_{-0.014-0.021}^{+0.010+0.023}$ & 3.026 & $3.028_{-0.014-0.025}^{+0.011+0.024}$ \\
\hline$n_{s}$ & 0.9712 & $0.9719_{-0.0039-0.0074}^{+0.0036+0.0072}$ & 0.9706 & $0.9720_{-0.0040-0.0076}^{+0.0038+0.0074}$ \\
\hline$\tau_{\text {reio }}$ & 0.05358 & $0.04963_{-0.0074-0.0096}^{+0.0041+0.010}$ & 0.05102 & $0.05158_{-0.0072-0.012}^{+0.0059+0.011}$ \\
\hline$w_{\mathrm{f}}$ & - & - & -0.9677 & $-0.9944_{-0.046-0.084}^{+0.041+0.089}$ \\
\hline$\beta$ & - & - & 0.9999 & $0.9997_{-0.00048-0.00990}^{+0.00047+0.00098}$ \\
\hline$z_{\text {reio }}$ & 7.502 & $7.084_{-0.69-1.0}^{+0.50+1.0}$ & 7.231 & $7.275_{-0.72-1.2}^{+0.59+1.2}$ \\
\hline$\Omega_{\mathrm{M}, 0}$ & 0.2871 & $0.2876_{-0.0044-0.0086}^{+0.0043+0.0086}$ & 0.2994 & $0.2945_{-0.0074-0.015}^{+0.0076+0.016}$ \\
\hline$H_{0}\left[\mathrm{~km} \mathrm{~s}^{-1} \mathrm{Mpc}^{-1}\right]$ & 69.56 & $69.54_{-0.36-0.71}^{+0.37+0.73}$ & 68.20 & $68.62_{-0.89-1.8}^{+0.79+1.7}$ \\
\hline$\sigma_{8}$ & 0.8079 & $0.8044_{-0.0051-0.0091}^{+0.0045+0.0096}$ & 0.7927 & $0.7956_{-0.0092-0.019}^{+0.0097+0.019}$ \\
\hline
\end{tabular}

In this analysis we use six likelihoods: The Planck likelihood with Planck 2018 data (containing high- $l$ TT,TE,EE, low- $l$ EE, low- $l$ TT, and lensing) (Aghanim et al. 2020), the Planck-SZ likelihood for the Sunyaev-Zeldovich effect measured by Planck (Ade et al. 2016, 2014), the CFHTLenS likelihood with the weak lensing data (Kilbinger et al. 2013; Heymans et al. 2013), the Pantheon likelihood with the supernovae data (Scolnic et al. 2018), the BAO likelihood with the baryon acoustic oscillations (BAO) data (Beutler et al. 2011; Anderson et al. 2014), and the BAORSD likelihood for BAO and redshift-space distortions (RSD) measurements (Alam et al. 2017; Buen-Abad et al. 2018).

Constraints on the cosmological parameters, considering the combined "Planck + Planck-SZ + CFHTLenS + Pantheon + BAO + BAORSD" data set, are displayed in Table 1. The triangle plot for selected cosmological parameters of TMG model is illustrated in Fig. 4. According to our results, TMG model predicts a lower value for the structure growth parameter $\sigma_{8}$ with respect to $\Lambda$ CDM model. So it seems that TMG model is more compatible with local measurements of structure growth, with a slight alleviation of $\sigma_{8}$ tension. Also the derived best fit value of $w_{\mathrm{f}}$ indicates that the fluid "f" has a negative pressure, and so we can conclude that it is a dark energy fluid. Interestingly enough, the best fit and the mean value of the fluid equation of state parameter demonstrate the quintessential character of dark energy, which is also effective in reducing the growth of structure. However, the phantom nature of $w_{\mathrm{f}}$ is also permitted within the $1 \sigma$ confidence level, where $-1.040<w_{\mathrm{f}}<-0.9532$. Moreover, according to the correlation between $\sigma_{8}$ and $H_{0}$, one can conclude that TMG model is not capable of providing a full reconciliation between low redshift probes and CMB measurements.

Furthermore, in order to understand which model provides a better fit to observational data, one can use the Akaike information criterion (AIC) defined as (Akaike 1974; Burnham \& Anderson 2002)

$\mathrm{AIC}=-2 \ln \mathcal{L}_{\max }+2 K$, 

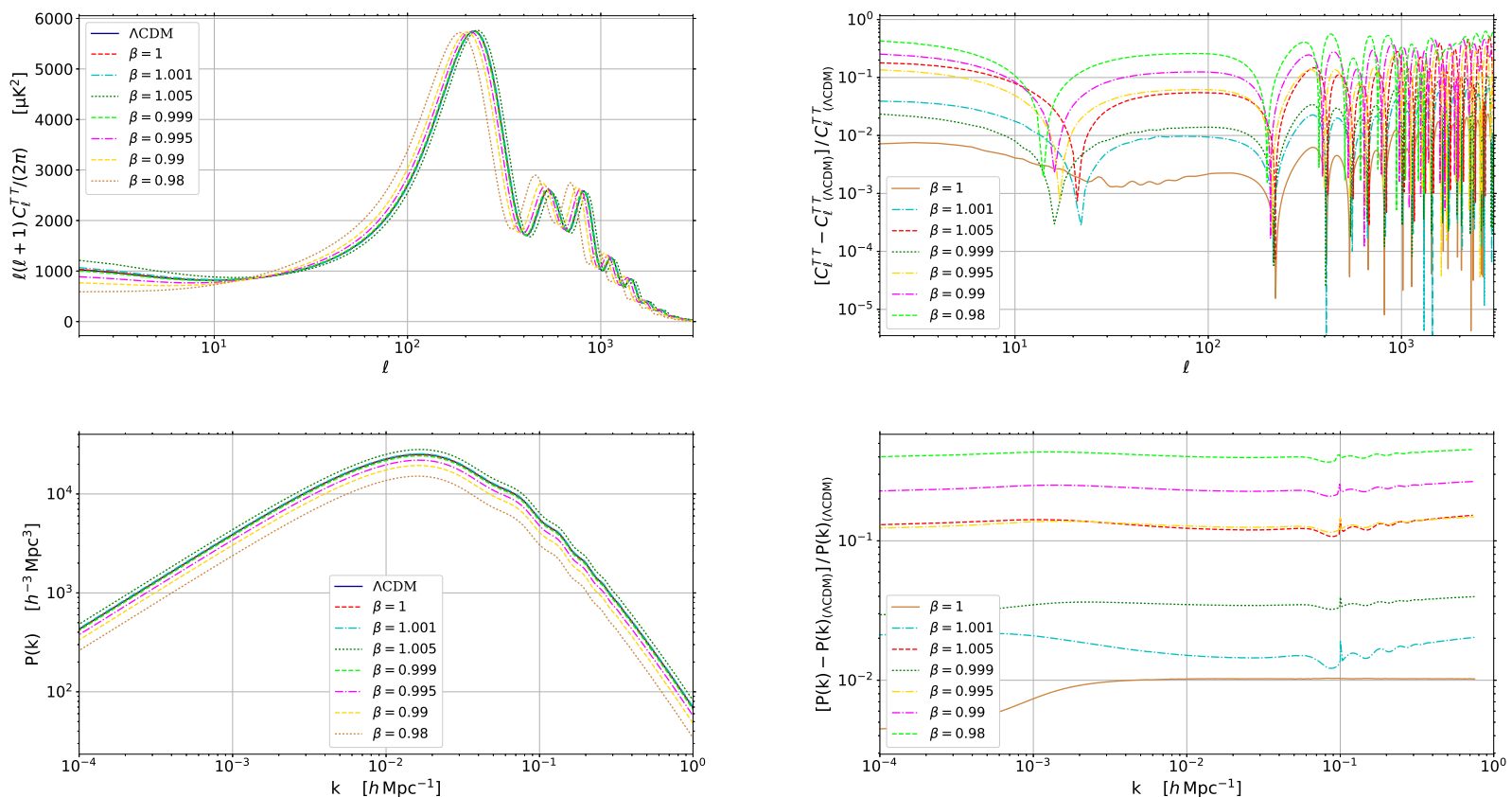

Figure 1. The CMB power spectra (upper left) and their relative ratio with respect to the standard model (upper right) for different values of $\beta$. Lower panels show the analogous diagrams for the matter power spectrum.

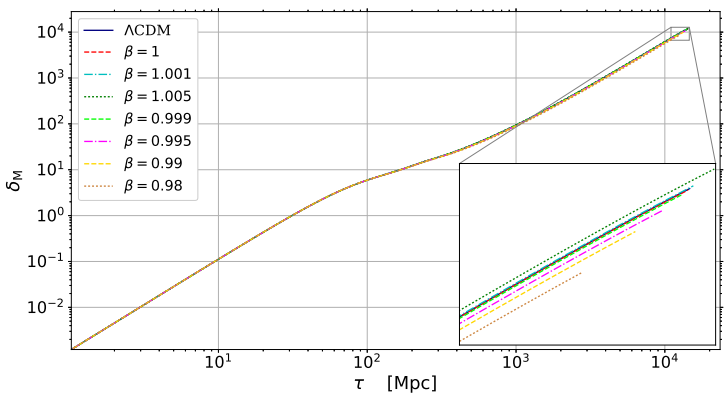

Figure 2. Matter density contrast diagrams in term of conformal time for TMG model with different values of $\beta$ compared to $\Lambda \mathrm{CDM}$.

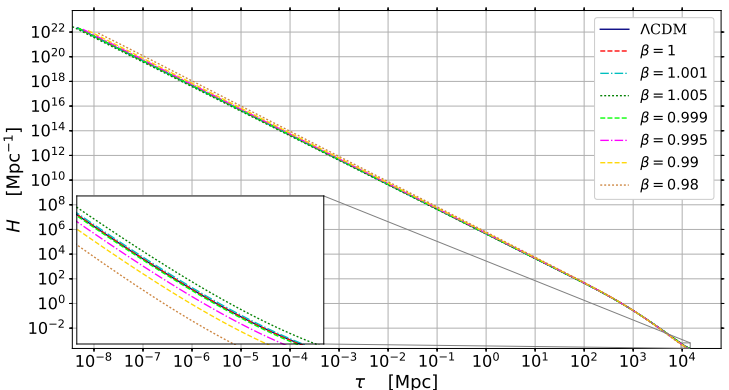

Figure 3. Hubble parameter in term of conformal time for TMG model with different values of $\beta$ compared to $\Lambda$ CDM.

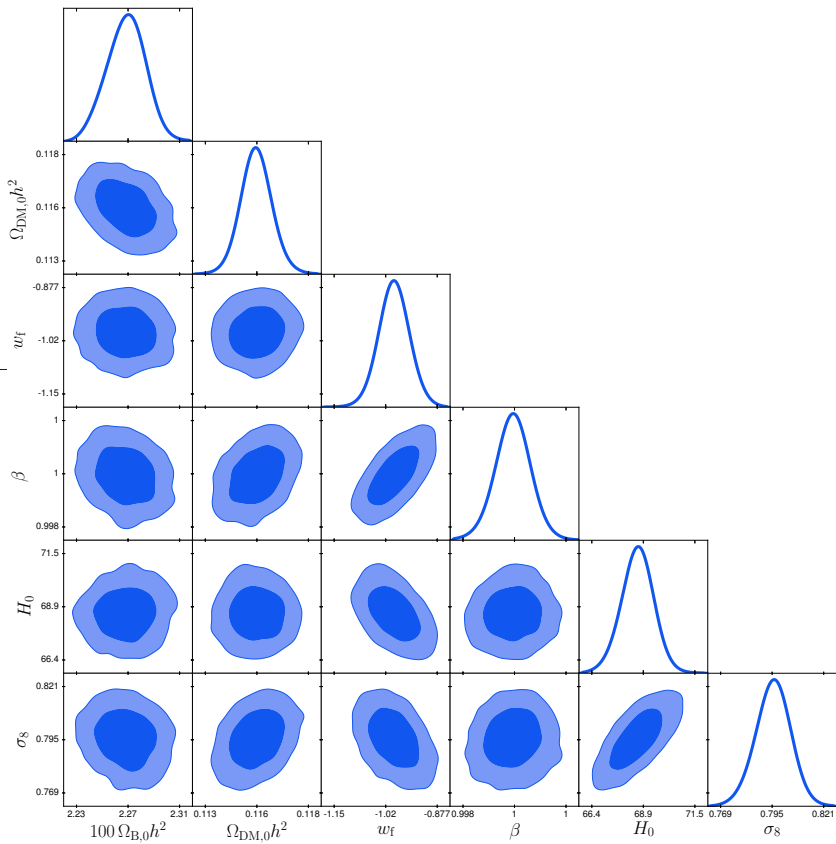

Figure 4. The one-dimensional posterior distribution and twodimensional posterior contours with $68 \%$ and $95 \%$ confidence limits from the "Planck + Planck-SZ + CFHTLenS + Pantheon + BAO + BAORSD" data set, for the selected cosmological parameters of TMG model.

in which $\mathcal{L}_{\max }$ is the maximum likelihood function, and $K$ is the number of free parameters. According to numerical analysis we have $\mathrm{AIC}_{(\Lambda \mathrm{CDM})}=3847.12$ and $\mathrm{AIC}_{(\mathrm{TMG})}=$ 3850.84 , which results in $\Delta \mathrm{AIC}=3.72$. So it can be concluded 
that the $\Lambda$ CDM model is more supported by observational data, while the TMG model can not be ruled out.

\section{CONCLUSIONS}

According to the analogy between thermodynamics and gravity, one can rewrite the Friedmann equation of FLRW universe in the form of the first law of thermodynamics at apparent horizon and vice versa. On the other hand, the Boltzmann-Gibbs (BG) theory is not convincing in gravitational systems which leads us to choose non-additive entropy to study such systems. In the present paper, we have considered the non-additive Tsallis entropy expression at apparent horizon of FLRW universe, to disclose its novel features on cosmological parameters. Accordingly we have derived the gravitational field equations when the entropy associated with the horizon is in the form of Tsallis entropy given in equation (2). In the cosmological background the obtained TMG leads to modified Friedmann equations describing the evolution of the universe. Employing numerical analysis, we constrain the cosmological parameters of this model with observational data, based on Planck CMB, weak lensing, supernovae, BAO, and RSD data. The obtained results indicate that the preferred value of $\sigma_{8}$ in TMG model is lower than the one in $\Lambda$ CDM, and so TMG model with a quintessential dark energy can slightly reduce the well known tension between the Planck CMB and local observations of $\sigma_{8}$.

\section{ACKNOWLEDGMENTS}

We are grateful to anonymous referee for valuable comments which helped us improve the paper significantly. We also thank Shiraz University Research Council.

\section{DATA AVAILABILITY}

No new data were generated or analysed in support of this research.

\section{REFERENCES}

Abdollahi Zadeh M., Sheykhi A., Moradpour H., Bamba K., 2018, The European Physical Journal C, 78, 940

Ade P. A. R., et al., 2014, Astronomy \& Astrophysics, 571, A20

Ade P. A. R., et al., 2016, Astronomy \& Astrophysics, 594, A24

Aghanim N., et al., 2020, Astronomy \& Astrophysics, 641, A6

Ai W.-Y., Hu X.-R., Chen H., Deng J.-B., 2013, Phys. Rev. D, 88, 084019

Akaike H., 1974, IEEE Transactions on Automatic Control, 19, 716

Akbar M., Cai R.-G., 2006, Physics Letters B, 635, 7

Akbar M., Cai R.-G., 2007a, Phys. Rev. D, 75, 084003

Akbar M., Cai R.-G., 2007b, Physics Letters B, 648, 243

Alam S., et al., 2017, Monthly Notices of the Royal Astronomical Society, 470, 2617

Allen S. W., Schmidt R. W., Fabian A. C., Ebeling H., 2003, Monthly Notices of the Royal Astronomical Society, 342, 287

Anderson L., et al., 2014, Monthly Notices of the Royal Astronomical Society, 441, 24
Ashtekar A., Baez J., Corichi A., Krasnov K., 1998, Phys. Rev. Lett., 80, 904

Audren B., Lesgourgues J., Benabed K., Prunet S., 2013, JCAP, 1302, 001

Bak D., Rey S.-J., 2000, Classical and Quantum Gravity, 17, L83

Banerjee R., Majhi B. R., 2008a, Physics Letters B, 662, 62

Banerjee R., Majhi B. R., 2008b, Journal of High Energy Physics, 2008, 095

Banerjee R., Majhi B. R., 2010, Phys. Rev. D, 81, 124006

Bardeen J. M., Carter B., Hawking S. W., 1973, Comm. Math. Phys., 31, 161

Bekenstein J. D., 1973, Phys. Rev. D, 7, 2333

Beutler F., et al., 2011, Monthly Notices of the Royal Astronomical Society, 416, 3017

Blas D., Lesgourgues J., Tram T., 2011, Journal of Cosmology and Astroparticle Physics, 2011, 034

Bousso R., 2005, Phys. Rev. D, 71, 064024

Brinckmann T., Lesgourgues J., 2018, MontePython 3: boosted MCMC sampler and other features (arXiv:1804.07261)

Buen-Abad M. A., Schmaltz M., Lesgourgues J., Brinckmann T., 2018, JCAP, 1801, 008

Burnham K., Anderson D., 2002, Model Selection and Multimodel Inference: A Practical Information-Theoretic Approach. Springer Verlag

Cai R.-G., Kim S. P., 2005, Journal of High Energy Physics, 2005, 050

Cai R.-G., Cao L.-M., Ohta N., 2010a, Phys. Rev. D, 81, 061501

Cai R.-G., Cao L.-M., Ohta N., 2010b, Phys. Rev. D, 81, 084012

Cai Y.-F., Liu J., Li H., 2010c, Physics Letters B, 690, 213

Calcagni G., 2005, Journal of High Energy Physics, 2005, 060

Danielsson U. H., 2005, Phys. Rev. D, 71, 023516

Das S., Majumdar P., Bhaduri R. K., 2002, Classical and Quantum Gravity, 19, 2355

Das S., Shankaranarayanan S., Sur S., 2008a, Black hole entropy from entanglement: A review (arXiv:0806.0402)

Das S., Shankaranarayanan S., Sur S., 2008b, Phys. Rev. D, 77, 064013

De Groot S., Mazur P., 1962, Non-equilibrium Thermodynamics. Dover books on physics and chemistry, North-Holland Publishing Company, https://books . google.ae/books?id=3b-wAAAAIAAJ

Eling C., Guedens R., Jacobson T., 2006, Phys. Rev. Lett., 96, 121301

Eune M., Kim W., 2013, Phys. Rev. D, 88, 067303

Frolov A. V., Kofman L., 2003, Journal of Cosmology and Astroparticle Physics, 2003, 009

Ghoshal A., Lambiase G., 2021, Constraints on Tsallis Cosmology from Big Bang Nucleosynthesis and Dark Matter Freeze-out (arXiv:2104.11296)

Gibbs J. W., 2010, Elementary Principles in Statistical Mechanics: Developed with Especial Reference to the Rational Foundation of Thermodynamics. Cambridge Library Collection - Mathematics, Cambridge University Press, doi:10.1017/CBO9780511686948

Hawking S. W., 1974, Nature, 248, 30

Hawking S. W., 1975, Communications in Mathematical Physics, 43, 199

Hendi S. H., Sheykhi A., 2011a, International Journal of Theoretical Physics, 51, 1125

Hendi S. H., Sheykhi A., 2011b, Phys. Rev. D, 83, 084012

Heymans C., et al., 2013, Monthly Notices of the Royal Astronomical Society, 432, 2433

Hinshaw G., et al., 2013, The Astrophysical Journal Supplement Series, 208, 19

Ho C. M., Minic D., Ng Y. J., 2010, Physics Letters B, 693, 567

Jacobson T., 1995, Phys. Rev. Lett., 75, 1260

Kaul R. K., Majumdar P., 2000, Phys. Rev. Lett., 84, 5255

Kilbinger M., et al., 2013, Monthly Notices of the Royal Astro- 
nomical Society, 430, 2200

Kiselev V. V., Timofeev S. A., 2011, Modern Physics Letters A, 26, 109

Konoplya R. A., 2010, The European Physical Journal C, 69, 555

Kothawala D., Sarkar S., Padmanabhan T., 2007, Physics Letters B, 652, 338

Ling Y., Pan W.-J., 2013, Phys. Rev. D, 88, 043518

Lyra M. L., Tsallis C., 1998, Phys. Rev. Lett., 80, 53

Ma C.-P., Bertschinger E., 1995, The Astrophysical Journal, 455, 7

Mamon A. A., Ziaie A. H., Bamba K., 2020, The European Physical Journal C, 80, 974

Mann R. B., Solodukhin S. N., 1997, Phys. Rev. D, 55, 3622

Myung Y. S., Kim Y.-W., 2010, Phys. Rev. D, 81, 105012

Nojiri S., Odintsov S. D., Paul T., 2021, Different faces of generalized holographic dark energy (arXiv:2105.08438)

Padmanabhan T., 2002, Classical and Quantum Gravity, 19, 5387

Padmanabhan T., 2005, Physics Reports, 406, 49

Padmanabhan T., 2010, Reports on Progress in Physics, 73, 046901

Padmanabhan T., 2012, Emergence and Expansion of Cosmic Space as due to the Quest for Holographic Equipartition (arXiv:1206.4916)

Padmanabhan T., Paranjape A., 2007, Phys. Rev. D, 75, 064004

Paranjape A., Sarkar S., Padmanabhan T., 2006, Phys. Rev. D, 74,104015

Percival W. J., et al., 2010, Monthly Notices of the Royal Astronomical Society, 401, 2148

Radicella N., Pavón D., 2010, Physics Letters B, 691, 121

Riess A. G., et al., 2016, The Astrophysical Journal, 826, 56

Riess A. G., et al., 2018, The Astrophysical Journal, 855, 136

Riess A. G., Casertano S., Yuan W., Macri L. M., Scolnic D., 2019, The Astrophysical Journal, 876, 85

Riess A. G., Casertano S., Yuan W., Bowers J. B., Macri L., Zinn J. C., Scolnic D., 2021, The Astrophysical Journal, 908, L6

Rovelli C., 1996, Phys. Rev. Lett., 77, 3288

Sadri E., 2019, The European Physical Journal C, 79, 762

Saridakis E. N., Bamba K., Myrzakulov R., Anagnostopoulos F. K., 2018, Journal of Cosmology and Astroparticle Physics, 2018, 012

Sayahian Jahromi A., Moosavi S. A., Moradpour H., Morais Graça J. P., Lobo I. P., Salako I. G., Jawad A., 2018, Physics Letters B, 780,21

Scolnic D. M., et al., 2018, The Astrophysical Journal, 859, 101

Sheykhi A., 2010a, The European Physical Journal C, 69, 265

Sheykhi A., 2010b, Phys. Rev. D, 81, 104011
Sheykhi A., 2012, International Journal of Theoretical Physics, 51, 185

Sheykhi A., 2018, Physics Letters B, 785, 118

Sheykhi A., 2020, The European Physical Journal C, 80, 25

Sheykhi A., Sarab K. R., 2012, Journal of Cosmology and Astroparticle Physics, 2012, 012

Sheykhi A., Teimoori Z., 2012, General Relativity and Gravitation, 44, 1129

Sheykhi A., Wang B., 2009, Physics Letters B, 678, 434

Sheykhi A., Wang B., Cai R.-G., 2007a, Phys. Rev. D, 76, 023515

Sheykhi A., Wang B., Cai R.-G., 2007b, Nuclear Physics B, 779, 1

Sheykhi A., Dehghani M., Hosseini S., 2013a, Physics Letters B, 726,23

Sheykhi A., Dehghani M., Hosseini S., 2013b, Journal of Cosmology and Astroparticle Physics, 2013, 038

Tsallis C., 1988, Journal of Statistical Physics, 52, 479

Tsallis C., 2011, Entropy, 13, 1765

Tsallis C., 2012, From nolinear statistical mechanics to nonlinear quantum mechanics - Concepts and applications (arXiv:1202.3178)

Tsallis C., Cirto L. J. L., 2013, The European Physical Journal C, 73,2487

Tsallis C., Mendes R., Plastino A., 1998, Physica A: Statistical Mechanics and its Applications, 261, 534

Tu F.-Q., Chen Y.-X., 2013, Journal of Cosmology and Astroparticle Physics, 2013, 024

Tu F.-Q., Chen Y.-X., 2015, General Relativity and Gravitation, 47,87

Verlinde E., 2000, On the Holographic Principle in a Radiation Dominated Universe (arXiv:hep-th/0008140)

Verlinde E., 2011, Journal of High Energy Physics, 2011, 29

Wang B., Abdalla E., Su R.-K., 2001, Physics Letters B, 503, 394

Wei H., 2010, Physics Letters B, 692, 167

Wei S.-W., Liu Y.-X., Wang Y.-Q., 2011, Communications in Theoretical Physics, 56, 455

Zhang J., 2008, Physics Letters B, 668, 353

da C. Nunes R., Barboza Jr. E. M., Abreu E. M. C., Neto J. A., 2014, Dark energy models through nonextensive Tsallis' statistics (arXiv: 1403.5706)

da Silva W. J. C., Silva R., 2021, The European Physical Journal Plus, 136, 543

This paper has been typeset from a $\mathrm{TE}_{\mathrm{E}} \mathrm{X} / \mathrm{L} \mathrm{T}_{\mathrm{E} X}$ file prepared by the author. 